Iranian Journal of Pathology | ISSN: 2345-3656

\title{
Histomorphological and Immunohistochemical Analysis of Pleural Neoplasms
}

\author{
Sandhya Venkatachala ${ }^{*}$, Swarna Shivakumar ${ }^{1}$, Meganathan Prabhu², Ramya Padilu ${ }^{2}$ \\ 1. Consultant Pathologist, Apollo Hospitals Bangalore, Bangalore, India \\ 2. Registrar Pathology, Apollo Hospitals, Bangalore, Bangalore, India
}

\begin{tabular}{c}
\hline KEYWORDS \\
\hline Lung neoplasms; \\
Mesothelioma; \\
Pleura; \\
Sarcoma; \\
Carcinoid \\
\hline
\end{tabular}

\section{Article Info}

Received 28 Jan 2017; Accepted 05 April 2018;

Published Online 17 July 2018;

\begin{abstract}
Background \& objective: Primary pleural neoplasms are rare entities compared with the pleural involvement by metastatic carcinoma.

The current study aimed at investigating the complete spectrum of pleural neoplasms and differentiating between them with the aid of immunohistochemistry (IHC).

Methods: Consecutive pleural biopsy specimens positive for a neoplasm, both primary and metastatic, were included in the study. Diagnosis or a differential diagnosis was suggested on histopathology confirmed by a panel of IHC markers such ascytokeratin(AE1/AE3), epithelial membrane antigen (EMA),vimentin, calretinin, CD34, CD99, SMA,bcl2, S100, CK7,CK20,TTF1,GCDFP, HMB45, LCA, synaptophysin, chromogranin, and naspsin.
\end{abstract}

Results: A total of 35 cases of pleural neoplasms included 15 (42.9\%) primary pleural neoplasms and $20(57.1 \%)$ metastatic carcinoma. Synovial sarcoma, malignant mesothelioma (MM), and solitary fibrous tumor (SFT) accounted for $14.2 \%, 11.4 \%$, and $8.5 \%$ of metastatic cases, respectively. Epithelioid sarcoma(ES), neuroendocrine carcinoma, and inflammatory myofibroblastic tumor were less common, each contributing to $2.9 \%$ of pleural neoplasms. Among the 20 cases of metastatic carcinoma, 13 were from the lung and 7 from the breast. Lung neoplasms metastasizing to the pleura were adenocarcinoma $(n=12)$ and atypical carcinoid $(n=1)$.

Conclusion: Analysis of histopathological pattern along with a panel of appropriate IHC markers distinguished the rare entities of pleural neoplasms essential to determine the prognosis and treatment modality.

Corresponding information:

Dr. Sandhya Venkatachala, Consultant Pathologist, Apollo Hospitals Bangalore, Bangalore, India E-mail : sandhyavenkatachala@yahoo.co.in

Copyright (C) 2018, IRANIAN JOURNAL OF PATHOLOGY. This is an open-access article distributed under the terms of the Creative Commons Attribution-noncommercial 4.0 International License which permits copy and redistribute the material just in noncommercial usages, provided the original work is properly cited.

\section{Introdudtion}

Metastatic carcinomas greatly outnumber primary neoplasms of the pleura including the diffuse malignant mesothelioma (MM). Metastatic involvement of the pleura is observed in lung cancer, lymphoma, breast and carcinomas of the female genital tract, and gastrointestinal tract in the descending order of frequency (1). A wide spectrum of lesions categorized as benign, low malignant potential, and malignant involved pleura. The benign lesions include the solitary fibrous tumor (SFT), adenomatoid tumor, multicystic mesothelioma, and calcifying fibrous tumor. Pleural thymoma, desmoid tumor, and the well differentiated papillary mesothelioma are neoplasms of low malignant potential (2). The pleura are infrequently involved in uncommon non-mesotheliomatous neoplasms such as synovial sarcoma, SFT, epithelioid sarcoma (ES), and inflammatory myofibroblastic tumor (2). Guniea DG and Erb CT further described other rare neoplasms as angiosarcoma, epithelioid hemangioendothelioma, and desmoplastic small round cell tumor (DSRCT) in their studies $(3,4)$. Pleuropulmonary blastoma is another extremely rare malignant pleural neoplasm with only two cases reported in the adults (2). Although rare, it is important to recognize 
these entities and differentiate them from the metastatic carcinoma and malignant mesothelioma since they have distinct treatment modality and prognosis (5). It is important to maintain a high index of suspicion to include these entities, while considering the differential diagnosis of a pleural tumor to ensure optimal treatment and prognosis. Diagnosis of malignant mesothelioma has legal implications (4). Literature search did not reveal any study encompassing both primary and secondary pleural neoplasms although case reports of the less common primary pleural neoplasms are present. Thus, the current study aimed at analyzing the complete spectrum of pleural neoplasms, both primary and metastatic, and differentiating between the entities with the aid of IHC.

\section{Material and Methods}

Consecutive pleural biopsy specimens positive for a neoplasm, both primary and metastatic were included in the study at a tertiary care hospital from January 2012 to October 2016. Diagnosis or a differential diagnosis was suggested on histopathology confirmed by IHC. The biopsies were subjected to a panel of IHC markers selected based on the morphology (2). The three major cell types were spindle cell, epithelioid/epithelial, and small blue cell. The IHC panel for neoplasms with spindle cells included cytokeratin, vimentin, calretinin, CD34, CD99, SMA, bc12 and S100; for epithelioid/epithelial cell neoplasms- cytokeratin, calretinin, epithelial membrane antigen (EMA), vimentin, CK7, CK20, TTF1, GCDFP, HMB45; and for small blue cell neoplasms including EMA, cytokeratin, LCA, synaptophysin, chromogranin, naspsin, and vimentin . For biphasic tumors the panel included cytokeratin, EMA, vimentin, calretinin, HMB45, CD34, CD99, SMA, bcl2, and S100 appropriate positive controls were run. Microwave antigen retrieval was done at $95^{\circ} \mathrm{C}(6)$. Ready to use Dako antibodies (vimentin- V9, EMA- E29, CK cocktail AE1/AE3, CK7 (OVTL12/30), CK20 (ITKs 20.8), TTF-1 (BGX-397A), napsinA (IP64), CK5/6 (D5/16B4), CD45-2B11, PD7/26/16, chromgraninA SP12, SMA-1A4, bc12-100, CD99-12E7, CD34 (QBEnd), calretinin (calret-1), synaptophysin SP-11, ER, PR, GCDFP, S100 (polyclonal Abs) were used.

\section{Results}

A total of 35 cases of pleural neoplasms included 15 (42.9\%) primary pleural neoplasms and $20(57.1 \%)$ metastatic carcinoma. The distribution of neoplasms is shown in Table 1.

Of the five cases of primary pleural synovial sarcoma, two were biphasic and three monophasic variants. The biphasic synovial sarcoma showed cleftlike glandular spaces amidst long cellular fascicles of spindle cells (Figure 1A).

The IHC pattern is shown in Table 2.

Table1 . The Spectrum of Pleural Neoplasms

\begin{tabular}{lcc}
\hline \multicolumn{1}{c}{ Pleural Neoplasm } & No. of Cases & Percentage \\
\hline Primary (15) & & \\
\hline Synovial sarcoma & 5 & 14.2 \\
Malignant Mesothelioma & 1 & 11.4 \\
The Ewing sarcoma & 1 & 2.9 \\
Neuroendocrine carcinoma & 3 & 2.9 \\
Solitary fibrous tumor & 1 & 8.5 \\
Inflammatory myofibroblastic tumor & & \\
\hline Metastatic carcinoma (20) & & \\
\hline & 12 & 2.9 \\
Pulmonary adenocarcinoma & 7 & 2.3 \\
Breast carcinoma & 1 & 2.9 \\
Atypical carcinoid & & \\
\hline
\end{tabular}


Positive staining for vimentin, Bcl2, CD99 (Figure $1 \mathrm{~B}, \mathrm{C}$ ) along with $\mathrm{CK} / \mathrm{EMA}$ in biphasic confirmed the diagnosis of synovial sarcoma. The monophasic variants were composed of spindle cells immunoreactive to $\mathrm{CK}$ and vimentin in addition to bcl 2 expression.

The four cases of mesothelioma (Figure 2A) consisted of three epithelioid (Figure 2C) and one des-

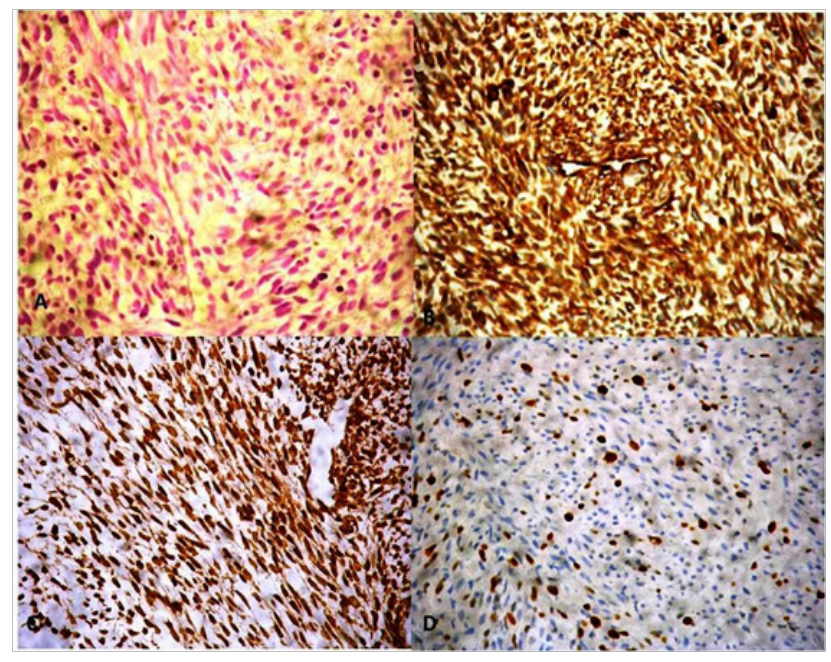

Figure 1. A, Biphasic synovial sarcoma; cleft-like space observed in the center, H\&E staining, 100X; B, CD99+ tumor cells, 100X; C, bcl-2 positive cells, 100X; D, ki67 expression, 100X moplastic variant (Figure 2D). The epithelioid variant showed cells with abundant eosinophilic cytoplasm, granular chromatin, and conspicuous nucleoli immunoreactive to calretinin (Figure 2B) and cytokeratin 7 and negative to epithelial markers-TTF1, CK20, and HMB45- as shown in Table 3.

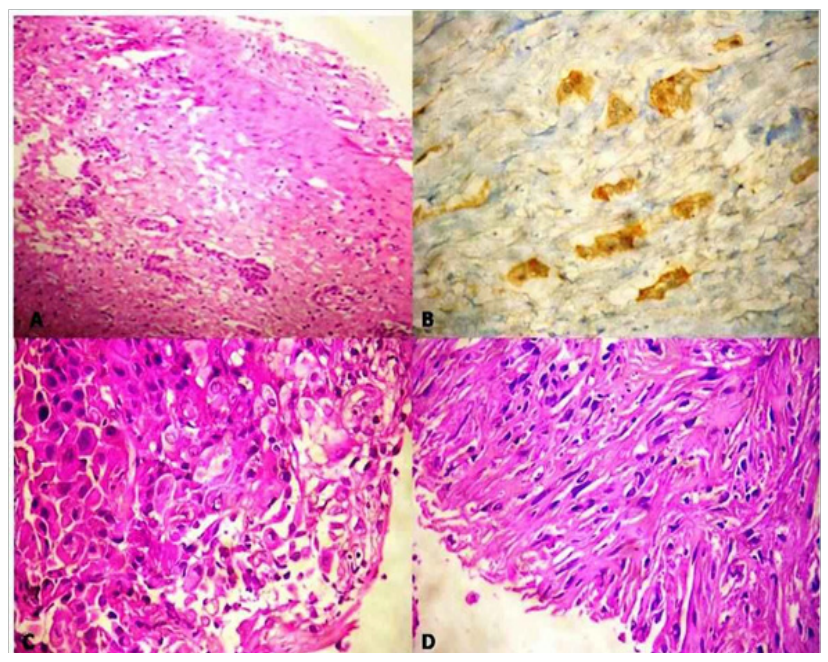

Figure 2. A, Nests of invasive mesothelial cells, H\&E staining, 40X; B, caletinin positivity in these cells, 100X; $\mathrm{C}$, Epithelioid mesothelioma cells with prominent nucleoli, H\&E staining, 100X; D, Atypical spindle cells in desmoplastic mesothelioma, H\&E staining, 100X

Table 2. IHC Staining Pattern in Synovial Sarcoma

\begin{tabular}{ccccccccccc}
\hline Synovial sarcoma(5) & CK & Vim & EMA & Bcl2 & CD99 & SMA & CD34 & Calretinin & S100 \\
\hline Monophasic(3) & 0 & 3 & 0 & 3 & 3 & 1 Focal expression & 0 & 0 & 0 \\
Biphasic (2) & 2 & 2 & 1 & 2 & 2 & 1 & 0 & 0 & 0 \\
\hline
\end{tabular}

Table 3. IHC Staining Pattern in Malignant Mesothelioma

\begin{tabular}{lccccccccc}
\hline $\begin{array}{l}\text { Malignant Mesothelioma } \\
\text { (4) }\end{array}$ & CK & CK7 & Vim & Calretinin & CD99 & SMA & CD34 & Bcl2 & S100 \\
\hline Epithelioid (3) & 2 & 2 & 2 & 3 & 0 & 0 & 0 & 0 & 0 \\
Desmoplastic (1) & 1 & 0 & 1 & 1 & 0 & 0 & 0 & Focal \\
\end{tabular}


199. Histomorphological and Immunohistochemical...

Vimentin expression varied. One other case was paucicellular and showed collagenous stroma with atypical invasive cells immunoreactive to calretinin and cytokeratin and, therefore, was designated as desmoplastic mesothelioma.

ES, composed of sheets of round to polygonal cells with fine granular chromatin, moderate amount of eosinophilic cytoplasm, and foci of necrosis (Figure 3A) expressing both cytokeratin and Vimentin were observed (Figure 3B,C). No expression of mesothelial markers was observed

The three cases of SFT showed cellular pattern with varying cellularity (Figure 4A). The tumor cells were positive for Vimentin, CD34 (Figure 4B), and CD99. Two of the cases also expressed bcl 2 and one of them SMA. Inflammatory myofibrobroblastic tumor (IMT) (Figure 4C) composed of spindle cells immunoreactive to SMA (Figure 4E) and round cells positive to CD68 constituted one of the cases in the current study. Few desmin positive cells were observed. Masson's trichome staining showed the myofibroblasts (Figure 4D).

Table 4 summarizes the IHC staining of SFT and IMT.

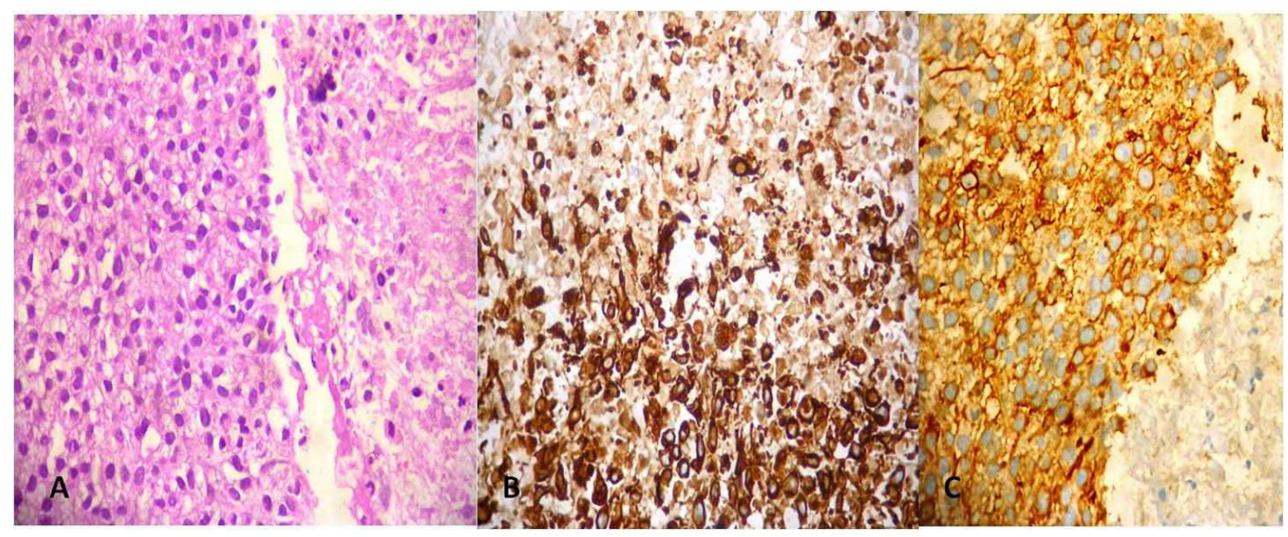

Figure 3. A, Epithelioid sarcoma with polygonal cells and necrosis, right half, H\&E staining, 100X; B, Cytokeratin positive tumor cells, 100X; C, vimentin positive tumor cells, 100X; Necrosis is observed in B and C.

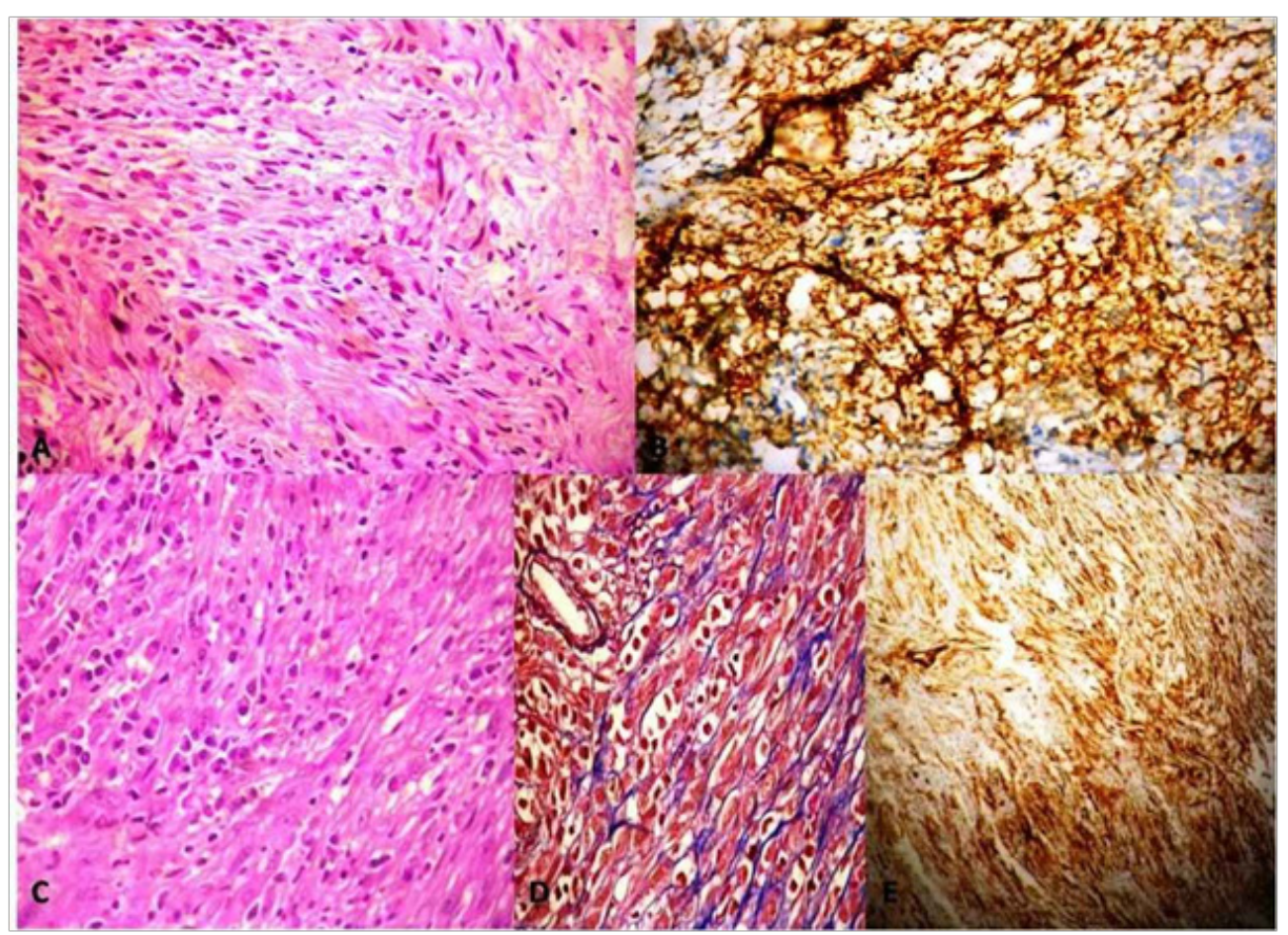

Figure 4. A, Solitary fibrous tumor with varying cellularity, 100X; B, CD34 positive cells in SFT, 100x; C, IMT with plasma cells (left upper half) and myofibroblasts (right half), H\&E staining, 100X; D, Masson's trichome staining, 400X; E, SMA+ IMT,100X 
Table 4. IHC Staining Pattern in Solitary Fibrous Tumor and Inflammatory Myofibroblastic Tumor

\begin{tabular}{cccccccccc}
\hline Neoplasm & CK & Vim & Calretinin & CD34 & CD99 & SMA & Bcl2 & S100 \\
\hline SFT(3) & 0 & 3 & 0 & 3 & 2 & 1 & 2 & 0 \\
IMT (1) & 0 & 1 & 0 & 0 & 0 & 1 & 0 & 0 \\
\hline
\end{tabular}

A single case of primary pleural malignant neuroendocrine tumor was observed (Figure 5A) positive for EMA, synaptophysin, chromogranin (Figure 5B), and negative for napsin, leucocyte common antigen, desmin, and CD99.

Proliferative index by immunohistochemical staining for Ki67 was 5\%. A lung/gastrointestinal (GI) primary was ruled out by fiberoptic bronchoscopy and endoscopic examination. Positron emission tomogra- phy-computed tomography (PET-CT) showed no occult primary tumor.

The 18 cases of metastatic pleural neoplasms are shown in Table 5 .

Pulmonary adenocarcinoma was the most common metastasis followed by infiltrating duct carcinoma of the breast (Figure 5C, D). One case of atypical carcinoid with $4 \mathrm{MF} / 10 \mathrm{HPF}$ metastatic to pleura was observed.

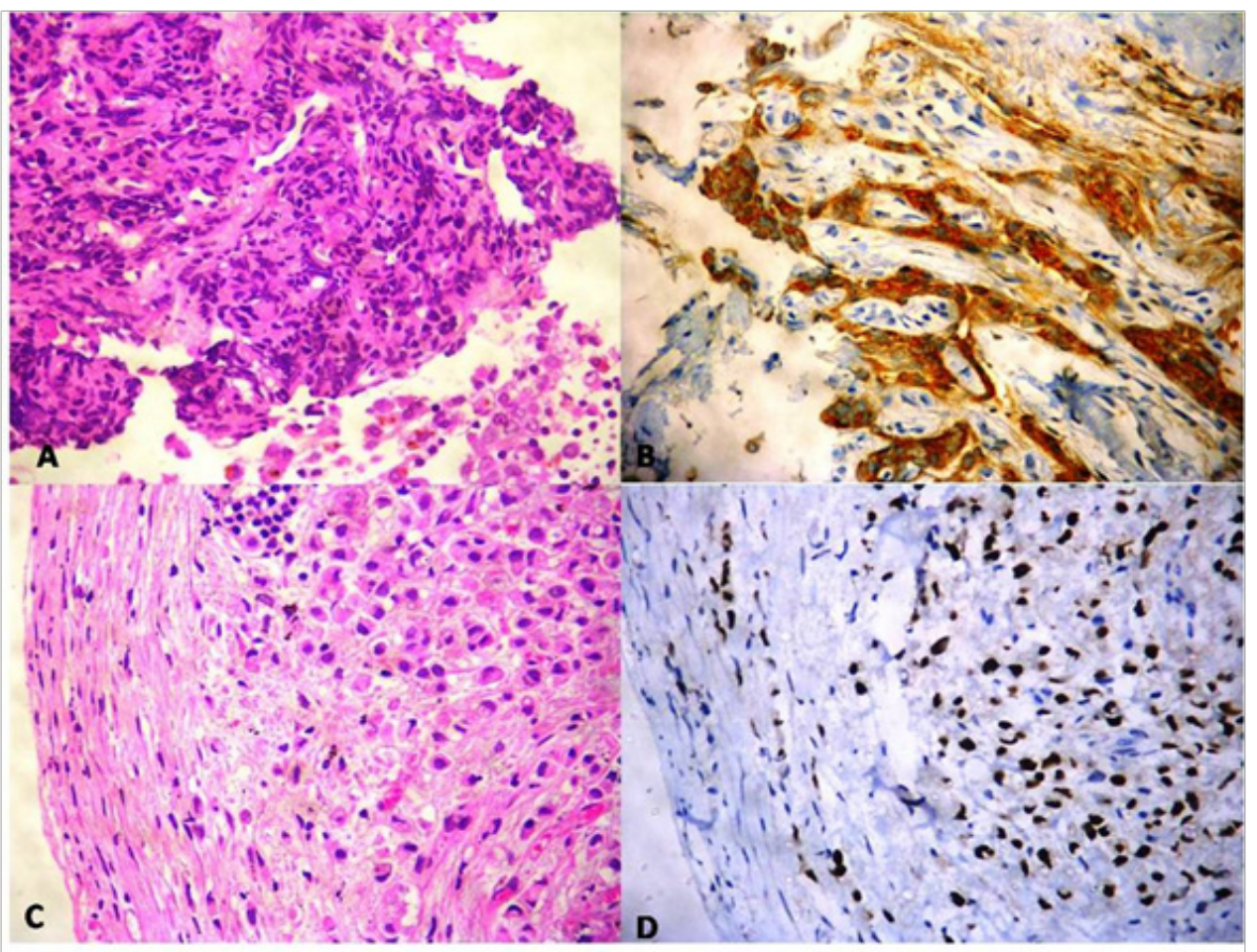

Figure 5. A, Cords of tumor cells in neuroendocrine carcinoma, H\&E staining, 100X; B, Chromogranin expression in these cells, 100X; C, Pleura with metastatic breast carcinoma cells, H\&E staining, 100X; D, ER expression in these cells, 100X

Table 5. Metastatic Pleural Neoplasms

\begin{tabular}{|c|c|c|}
\hline Primary Neoplasm & No. of Cases & IHC Profile \\
\hline Pulmonary adenocarcinoma & 12 & $\mathrm{CK} 7+, \mathrm{TTF} 1+$, napsin + calretinin- \\
\hline Infiltrating duct carcinoma breast & 7 & $\mathrm{CK} 7+, \mathrm{ER}+, \mathrm{PR}+, \mathrm{GCDFP}+, \mathrm{TTF}-$ calretinin- \\
\hline Atypical pulmonary carcinoid & 1 & $\begin{array}{c}\mathrm{CK} 7+, \mathrm{CK} 5 / 6+\text {, chromogranin }+ \text {, synaptophysin }+ \text {, } \\
\text { and napsin- }\end{array}$ \\
\hline
\end{tabular}


201. Histomorphological and Immunohistochemical...

\section{Discussion}

Metastatic pleural neoplasms are more common compared with the primary pleural neoplasms $(1,2)$. The same was observed in the current study with $42.9 \%$ of primary neoplasms compared with $57.1 \%$ of secondary pleural tumors. A wide variety of uncommon primary pleural neoplasms were encountered. These entities need to be identified, which often requires a panel of IHC markers in addition to light microscopy. Primary pleural neoplasms are first discussed followed by metastatic tumors.

Synovial sarcoma was the most common primary pleural neoplasm (14.2\%) slightly outnumbering malignant mesothelioma (11.4\%) in the current study contrary to other reports (7). Only 17 cases of primary synovial sarcoma of pleura are reported to date $[8,9,10]$ with equal incidence of monophasic and biphasic variants, mean age of presentation being 40 years (ranged 15-60) and slight male preponderance $(57 \%)$ (8). Similar findings were observed in the current study with $80 \%$ incidence in males, mean age of 43 years, and 2:3 ratio of monophasic: biphasic variants. Differential diagnosis of synovial sarcoma (SS) includes sarcomatoid MM, SFT with its malignant variant, malignant peripheral nerve sheath tumor (MPNST), metastatic spindle cell melanoma for the monophasic variant, and a biphasic malignant mesothelioma for the biphasic variant. Compact cellular long spindles as observed in the current study differentiate SS from sarcomatoid MM. Other features include the presence of mast cells and PAS+ (diastase resistant), mucicarmine + (hyaluronidase resistant) secretions in biphasic SS, but not in biphasic MM (10). Immunoreactivity to bcl-2, and CD99 with focal cytokeratin reactivity in the epithelial component as observed in the current study readily differentiate it from MM, which exhibits diffuse positivity for CK and calretinin. Diagnostic utility is another marker for WT1 expressed only in the latter (10). In difficult cases, with overlapping features, the diagnosis is by demonstration of chromosomal translocation $\mathrm{t}(\mathrm{x} ; 18)$ (p11.2; q11.2) in SS. The cellular variant of SFT morphologically resembles SS. CD34 immunoreactivity in SFT clinches the diagnosis. However, the malignant SFT defined by the presence of nuclear atypia, necrosis, hypercellularity, and $>4$ mitoses $/ 10 \mathrm{HPF}$ can be negative for CD34. The absence of these features differentiates SS from malignant SFT. Prominent nucleoli with HMB45 positivity differentiate melanoma from SS. A negative S100/GFAP rules out MPNST.

Malignant mesothelioma was the second most common category. All the patients were elderly males with history of environmental exposure to asbestos as reported in the literature (11). Non-asbestos fibre erionite was also associated with mesothelioma in Turkey (11). Epthelioid mesothelioma outnumbered the desmoplastic variant, similar to other studies $(11,12)$. Immunoreactivity to calretinin and negativity to HMB45 and other epithelial markers ruled out the possibility of metastatic melanoma, carcinoma. The differentiation of malignant mesothelioma from SS is already discussed. The single case of desmoplastic variant showed dense collagenous stroma with atypical mesothelial cells lining the haphazardly arranged slit-like spaces satisfying the 2004 WHO (the World Health Organization) criteria for desmoplastic mesothelioma. Fibrinous pleurisy needs to be differentiated from this variant. $\mathrm{MM}$ is an aggressive disease with a median survival of 12 to 18 months and epithelioid types have better prognosis than the sarcomatoid/ desmoplastic variants. Unusual patterns of MM such as signet ring cell mesothelioma, lymphocytoid type, deciduoid, and mesothelioma with heterologous elements need to be identified as they mimic signet ring cell carcinoma, lymphoma, metastatic trophoblastic disease, and sarcoma (11).

ES is a rare neoplasm accounting for less than $1 \%$ of soft tissue sarcoma (13). Primary ES of the pleura is extremely rare with only two case reports published so far $(14,15)$. ES are of two types, conventional distal and proximal type (15). Unlike the distal type, which occurs in distal extremities, the proximal type is observed in perineum, genital tract, head, and trunk. Presence of necrosis and polygonal to spindle cells are common features. The absence of granulomas and the frequent presence of rhabdoid cells are unique to 
the proximal type (15). The single case of ES showed polygonal cells in sheets with necrosis with expression of $C K$ and vimentin. No granulomas or rhabdoid cells were observed. ES is immunoreactive to $\mathrm{CK}$ and vimentin (15).Immunostaining for INI 1(tumor suppressor gene) is used to confirm the diagnosis as a complement to the IHC markers.

SFT accounts for less than $5 \%$ of all pleural tumors and $10 \%-30 \%$ of these tumors are malignant (16). Three patterns are described: patternless pattern of Stout, hypocellular tumor with ropey collagen and few spindle cells in slit-like spaces; Hemangio-pericytomatous pattern; and storiform pattern spindle cell (2).The three cases in the current study showed the cellular pattern. Positivity for CD34, bcl2 with Vimentin characterises the tumor as seen in the present study (17). Zhu Y et al., reported a few CD34 negative cases of SFT (17). SMA expression as observed in one of the current study cases is reported by Martorell (18). The criteria to diagnose malignant SFT include: hypercellularity, > $4 \mathrm{MF} / 10 \mathrm{HPF}$, hemorrhage, and necrosis. They have poor prognosis, unlike SFT (16).

IMT is an intermediate biological potential neoplasm, which can recur and infrequently metastasize, accounting for less than $1 \%$ of pulmonary tumors. Pleural involvement is described as an extension of pulmonary IMT (19). Exclusive pleural IMT is extremely rare, with three cases reported in the literature $(19,20,21)$, characterized by the presence of myofibroblasts and chronic inflammatory cells, the tumor is positive for SMA and vimentin, and negative for CD34 as observed in the current study case; CD68 expression was also observed. $A L K$ gene is expressed in $50 \%$ of IMT cases and the other $50 \%$ are malignant (20).

A single case of primary malignant neuroendocrine tumor of pleura is reported (21) so far in the literature. This rare tumor was observed in the current study, immunoreactive to chromogranin and synaptophysin. PETCT ruled out an occult primary similar to the case reported by Das and Pratap (21). Immunonegativity for LCA, CD99, and desmin ruled out other small blue cell tumors such aslymphoma, the Ewing/PNET, and DSRCT, respectively.

Metastatic pleural tumors are more common malignant neoplasms of pleura, lungs, breast, and hematologic malignancy (lymphoma) are the common primary metastasizing neoplasms (1). In the current study, pulmonary adenocarcinoma was the most common followed by breast. One case of metastatic neuroendocrine tumor from an atypical bronchial carcinoid was observed. Pleural metastasis from bronchial carcinoids is rare as described by Klee $\mathrm{H}$ and others (22).

Recognition of this spectrum of uncommon primary pleural neoplasms and differentiating them from each other is essential, since they have distinct treatment and prognosis as in malignant mesothelioma (sarcomatoid/desmoplastic) vs synovial sarcoma (monophasic variant). SS is responsive to chemotherapy, has longer survival (five-year disease free period of $20.9 \%$ ) while MM has an average survival of less than 12 months and is chemoresistant, especially sarcomatoid variant $(7,10)$. In addition, a diagnosis of MM has legal implications due to its strong association with asbestos exposure.

Assessment of histological pattern along with a panel of appropriate IHC markers clinches the final diagnosis. Thus, the panel of markers used in the current study- cytokeratin, calretinin, EMA, vimentin, CK7, CK20, TTF1, GCDFP, and HMB45 for epithelial/epithelioid neoplasms, cytokeratin, vimentin, calretinin, CD34, CD99, SMA, bcl2, and S100 for spindle cell neoplasms, and EMA, cytokeratin, LCA, synaptophysin, chromogranin, naspsin and vimentin for small blue cell tumors provide sufficient clarity and distinction among the rare entities. In difficult situations, however, cytogenetic study and EM are needed to make a definitive diagnosis.

\section{Acknowledgement}

The authors wish to thank Amul Rani for her technical support.

\section{Conflict of Interests}

Authors declared no conflict of interests. 


\section{References}

1. Agrawal A, Tandon R, Singh L, Chawla A. Clinico-pathological profile and course of malignant pleural effusion in a tertiary care teaching hospital in western UP with special reference to lung cancer. Lung India. 2015;32(6):678-9. PMID:26664199

2. Granville L, Laga AC, Allen TC, Dishop M, Roggli VL, Churg A et al. Review and update of Uncommon Primary pleural tumors . A practical approach to diagnosis. Arch Pathol Lab Med. 2005;129(11):1428-43. PMID:16253024

3. Erb CT, Johnson KM, Kim AW. Rare pleural tumors. Clin Chest Med. 2013;34(1):113-

36. $\quad$ https://doi.org/10.1016/j.ccm.2012.12.001 PMID:23411062

4. Donald G Guinee Timothy Craig Allen Primary pleural neoplasia: Entities other than diffuse malignant mesothelioma. Arch Pathol Lab Med. 2008;132(7):1149-70. PMID: 18605768

5. Zandwijk NV, Clarke C, Henderson D, Musk AW , Fong K, Nowak A et al. Guidelines for the diagnosis and treatment of malignant pleural mesothelioma. J Thorac Dis. 2013;5(6):254307.

6. Jackson P, Blythe D. Immunohistochemical techniques. In: Bancroft JD, Gamble M. Theory and Practice of histological techniques.6nd ed. China: Elsevier; 2008. p.465.

7. Klebe S, Brownlee NA, Mahar A, Bruchette JL, Sporn TA, Vollmer RT. Sarcomatoid mesothelioma: aclinico-pathologic correlation of 326 cases. Mod Pathol. 2010;23(3):470-9. https://doi. org/10.1038/modpathol.2009.180 PMID:20081811

8. Ng SB, Ahmed Q, Tein SL, Sivaswaren C, Lau LC. Primary pleural Synovial sarcoma. A case report and review of literature. Arch Pathol Lab Med. 2003;127(1):85-90. PMID:12521374

9. Loscertales J, Trivino A, Gallardo G, Congregado M. Primary monophasic synovial sarcoma of the pleura; diagnosis and treatment. Interact Cardiovasc Thorac Surg. 2011;12(5):885-

7. https://doi.org/10.1510/icvts.2010.259531 PMID:21324917

10. Mardi K, Chauhan P, Kaushal V. Primary synovial sarcoma of pleura: A case report and re- view of the literature. Clin Cancer Investig J. 2016;5(5):59-62.

11. Smith M, Colby T. The diagnosis of thoracic malignant mesothelioma: Practical considerations and Recent developments. Turk Patoloji Derg. 2014;30(1):1-10.

12. Allen TC, Cagle PT, Churg AM, Colby TV, Gibbs AR, Hammar SP. Localised malignant mesothelioma. Am J Surg Pathol. 1994;18(4):357-63.

13. Chbani L, Guillou L, Terrier P, Decoulaere AV, Gregoire F, Lacombe MJet al.Epithelioid sarcoma- A clinicopathologic and Immunohistochemical Analysis of 106 cases from the French Sarcoma Group.Am J Clin Pathol. 2009;131(2):222-7. https://doi.org/10.1309/AJCPU98ABIPVJAIV PMID:19141382

14. Madsen GA, Rasmussen TR, Baerentzen S. A case of pleural epithelioid sarcoma of proximal type presenting as malignant pleural mesothelioma. J Thorac Oncol. 2013;8(1):89-90. https://doi.org/10.1097/JTO.0b013e31829e7f16 PMID:24457248

15. Panagiotopoulos N, Zapata S, Rass1 D, Coonar A, Scarci M. Primary epithelioid sarcoma of the pleura: An intricate diagnosis. Ann Thorac Surg. 2013;96(5):e79. https://doi.org/10.1016/j. athoracsur.2013.05.107 PMID:23992738

16. Jeon HW, Kwon SS, Kim YD. Malignant Solitary fibrous tumor of the pleura slowly growing over 17 yrs: case report. J Cardiothorac Surg. 2014;9(1):113. PMCID:PMC4078939

17. Zhu Y, Du K, Ye X, Song D, Long D.Solitary fibrous tumor of pleura and lung:report of twelve cases. J Thorac Dis. 2013;5(3):310-3. PMID:23825765

18. Martorell M, Valles AP, Gozalbo F, Garcia JA, Gutierrez J, Goana J. Solitary fibrous tumor of the thigh with epithelioid features: a case report. Diagn Pathol. 2007;2(1):19. https://doi. org/10.1186/1746-1596-2-19 PMID:17577399 PMCID:PMC1913496

19. Nobre de Jesus G, Rocha SL, Lopes JM, Santos JM, Oliveira PS, Victorino RM. Inflammatory Myofibroblastic Tumour: Report of a Rare Form with Exclusive Pleural Involvement. Case Rep Pulmonol. 2014;2014:621941. PMID:25525549 
20. Ueno T, Yamashita M, Sawada S, Suehisa H, Kawamoto H, Takahata H. A rare case of Inflammatory myofibroblastic tumor of the diaphragmatic parietal pleura with dissemination. Acta Med Okayama. 2015;69(1):65-8. PMID:25703173

21. Das A, Pratap A. Primary Malignant Neuro- endocrine Tumour of Pleura: First Case Report. Case reports in oncological medicine. 2016;2016. https://doi.org/10.1155/2016/5462380

22. Klee H, Vestring T, Bittmann I. Pleural metastasis of a typical carcinoid 7yrs after lobectomy. Pneumologie. 2008;62(10):607-10. https://doi. org/10.1055/s-2008-1038226 PMID: $\underline{18711695}$

\section{How to Cite This Article}

Venkatachala S, Shivakumar S, Prabhu M, Padilu R. Histomorphological and Immunohistochemical Analysis of Pleural Neoplasms. Iranian Journal of Pathology, 2018; 13(2): 196-204. 